Revue trimestrielle sur l'image géographique et les formes du territoire

$131 \mid 2021$

Varia

\title{
Solhan : cartographier le terrorisme et la dynamique territoriale d'une insurrection
}

Hervé Théry et Daniel Dory

\section{OpenEdition}

Journals

Édition électronique

URL : https://journals.openedition.org/mappemonde/6129

DOI : $10.4000 /$ mappemonde.6129

ISSN : 1769-7298

Éditeur

UMR ESPACE

Référence électronique

Hervé Théry et Daniel Dory, « Solhan : cartographier le terrorisme et la dynamique territoriale d'une insurrection », Mappemonde [En ligne], 131 | 2021, mis en ligne le 08 juillet 2021, consulté le 14 juillet 2021. URL : http://journals.openedition.org/mappemonde/6129; DOI : https://doi.org/10.4000/ mappemonde.6129

Ce document a été généré automatiquement le 14 juillet 2021.

\section{cc) (1)}

La revue Mappemonde est mise à disposition selon les termes de la Licence Creative Commons Attribution - Pas d'Utilisation Commerciale - Partage dans les Mêmes Conditions 4.0 International. 


\title{
Solhan : cartographier le terrorisme et la dynamique territoriale d'une insurrection
}

\author{
Hervé Théry et Daniel Dory
}

1 Dans la nuit du 4 au 5 juin 2021 un groupe (encore) non identifié a massacré environ 160 personnes dans la localité de Solhan, au nord-est du Burkina Faso. Cette attaque est la plus meurtrière qu'ait connu ce pays depuis sa création et, en particulier, depuis l'implantation du djihadisme violent sur son territoire vers 2015. Elle s'est, en outre, produite au moment où les auteurs de cette note venaient de terminer l'élaboration d'une analyse cartographique du terrorisme dans la zone des «Trois frontières » (Mali - Niger - Burkina Faso) ${ }^{1}$. Il nous a donc paru intéressant de poursuivre ce travail en y incluant cet épisode, afin de contribuer à une meilleure compréhension des dynamiques territoriales du processus insurrectionnel en cours dans cette région du Sahel Ouest Africain. Après une rapide actualisation «à chaud» de notre carte ${ }^{2}$, nous nous proposons maintenant d'approfondir la réflexion sur les possibilités offertes par l'outil cartographique pour comprendre la logique spatiale en jeu dans ce cas.

2 Pour ce faire, nous évoquerons brièvement quelques éléments contextuels, avant de fournir des références sur l'état actuel de la recherche en géographie et cartographie du terrorisme ; enfin les sources et la méthodologie de notre carte seront exposées, en insistant sur leur intérêt pour contribuer à la formulation d'hypothèses prédictives.

\section{Une dynamique conflictuelle ancienne réactivée par le djihadisme hybride}

3 Les tout récents évènements de Solhan s'inscrivent dans une longue suite de conflits localisés dont les aspects ethnopolitiques et religieux renvoient à des enjeux structurels de disputes pour des ressources rares (eau et pâturages, surtout) dans un environnement semi-aride confronté à une expansion démographique incontrôlée. La difficulté à gérer ces antagonismes, par exemple entre agriculteurs sédentaires et 
pasteurs nomades, par des moyens non violents est d'autant plus grande que les mécanismes traditionnels de distribution du pouvoir dans les États concernés ont été remplacés par les procédés formels de la «démocratie» qui transforment inévitablement les élections en opérations de comptage ethnique. Dans ces conditions on comprend sans mal l'attrait que représente l'islamisme djihadiste qui permet de consolider des alliances trans-ethniques sur la base de réseaux parfois internationaux de solidarité et d'échanges divers. Si l'on ajoute à cette toile de fond la présence croissante, depuis au moins deux décennies, de réseaux criminels se livrant à divers trafics (drogue, contrebande, migrants, etc.) dans et au travers de la zone sahélienne, on parvient à un début de compréhension de la situation géopolitique actuelle, par ailleurs bien décrite dans plusieurs publications de synthèse ${ }^{3}$.

Dans ces conditions, s'il est difficile d'attribuer l'attaque de Solhan à un acteur en particulier (filiale d'al Qaïda ou de l'État Islamique, milice ethnique et/ou entité criminelle, ou un mélange instable de plusieurs de ces catégories), il est, en revanche, possible de cerner (et de cartographier) une vaste zone où se déploient diverses formes de violence, notamment terroriste. Pour en rendre compte, on dispose d'un socle de connaissances en matière de géographie du terrorisme qui peut être mobilisé pour soutenir la recherche cartographique sur la question.

\section{La géographie du terrorisme : un chantier prometteur}

5 Même si la recherche géographique est encore assez marginale au sein du champ des études sur le terrorisme, plus connues sous leur désignation anglaise de terrorism studies $^{4}$, on dispose déjà d'un nombre non négligeable de travaux qui s'attachent à étudier la distribution spatiale des actes terroristes, la territorialité des acteurs qui recourent à cette forme de violence (principalement) politique, ainsi que celle de plusieurs autres composantes du complexe terroriste ${ }^{5}$. De même, plusieurs publications incluent des représentations cartographiques de la dynamique territoriale du djihadisme en Afrique de l'Ouest, permettant de visualiser sa progression vers le sud ${ }^{6}$. Ce que nos cartes confirment à partir de données concernant la localisation des actes terroristes répertoriés dans la Global Terrorism Database (GTD).

\section{Une carte exploitant la GTD par le calcul des barycentres}

6 La GTD répertorie les évènements considérés comme relevant du terrorisme dans le monde depuis 1970. Elle est basée à l'Université du Maryland, et elle est régulièrement alimentée par le consortium START qui bénéficie d'un soutien direct du gouvernement des États-Unis. Ses données, issues principalement d'informations de presse sur les incidents, ont été synthétisées depuis 2012 dans le Global Terrorism Index, rapport élaboré en collaboration avec l'Institute for Economics and Peace (Sidney). Actuellement, il s'agit de la meilleure base de données disponible, incluant tant les incidents domestiques que ceux qui relèvent du terrorisme international ${ }^{7}$. Cependant, tout comme les autres sources similaires, sa fiabilité dépend de la valeur de ses définitions, des procès de codification, des lacunes dans le recueil ou la disponibilité des informations, etc. ${ }^{8}$ Il en résulte que lorsque l'on travaille, comme nous l'avons fait ici, 
sur des données agrégées de la GTD, telles que l'on peut les décharger du site du consortium (https://www.start.umd.edu/gtd/), sans pouvoir filtrer les cas douteux ou clairement non terroristes, les cartes obtenues sont à envisager plus comme des indications de tendances et de distributions générales, que comme la représentation exacte d'un phénomène dont les contours sont parfaitement maîtrisés.

Ce média ne peut être affiché ici. Veuillez vous reporter à l'édition en ligne http:// journals.openedition.org/mappemonde/6129

L'histogramme (figure 2), permet de distinguer la spécificité locale des deux premiers épisodes, et la croissance spectaculaire de la violence (notamment terroriste) à partir de 2012.

Figure 2. Périodisation des actes terroristes

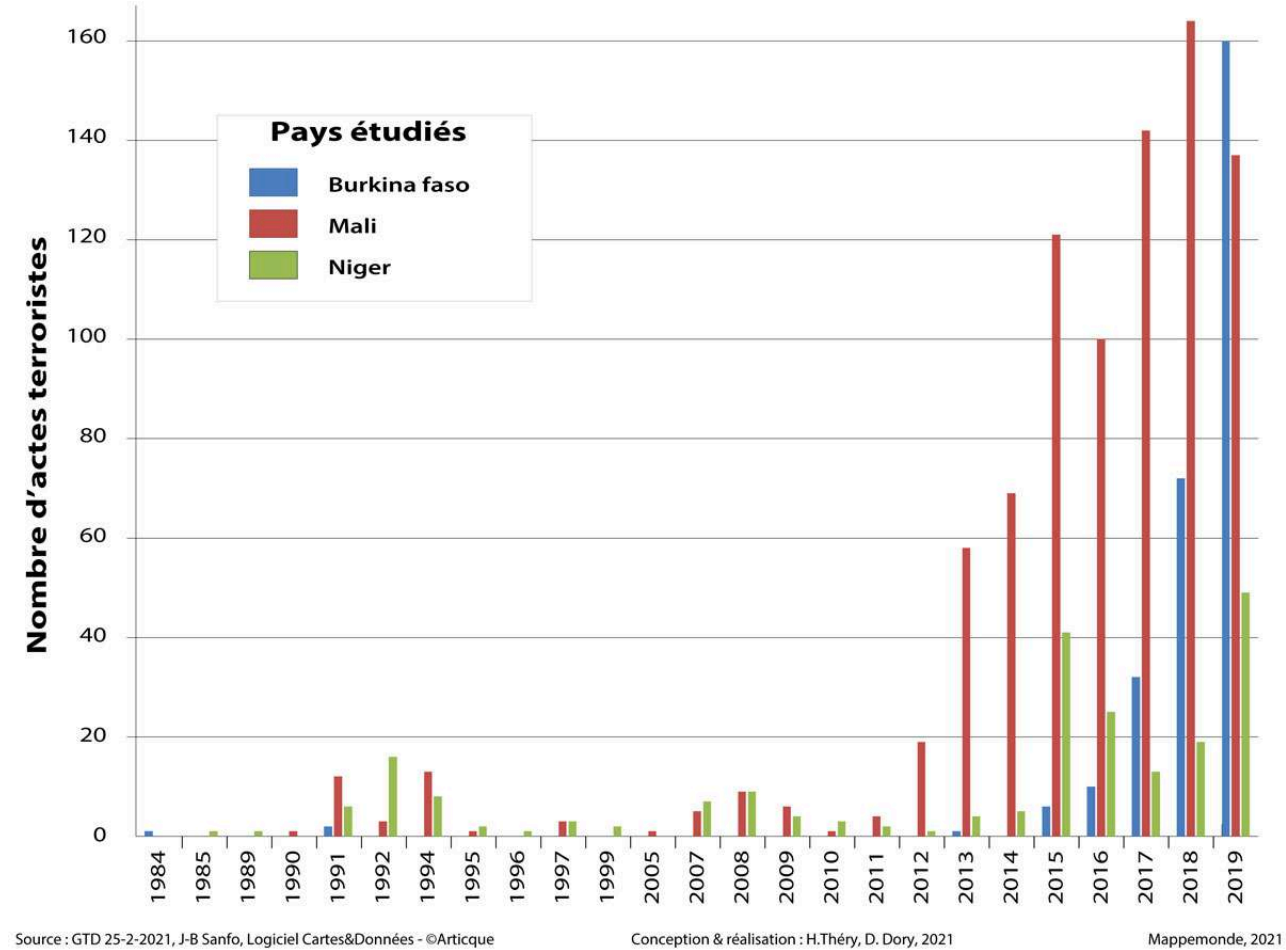

Des cercles proportionnels indiquent le nombre d'actes recensés en chaque lieu, identifié dans la base en latitude et longitude, donc sans les contraintes de la maille administrative. Leur couleur est donnée par la période au cours de laquelle ils ont été commis, la discrétisation ayant été faite au vu de l'histogramme pour distinguer : a) le milieu de la révolte touarègue au Niger et Mali (1990-1996) ; b) la révolte touarègue suivante (2007-2009) et ses suites; c) les années immédiatement antérieure et postérieure au début de l'opération Serval (2013) ; d) la dynamique de la conflictualité dans les années suivantes (2019 : dernières données disponibles).

Pour mieux voir le déplacement de la violence au cours du temps nous avons eu recours au calcul des barycentres (les centres de gravité pondérés de l'ensemble des attaques de chaque période), l'un des modules du logiciel de cartographie utilisé, Cartes et Données (Articque). 

direction du Burkina Faso au cours des dernières années et la tendance à la transnationalisation que le djihadisme introduit dans la zone, dont le foyer au sud-est du Niger (lié à Boko Haram) est une autre expression.

va plus loin en tentant de mettre en rapport les concentrations d'actes terroristes dans certaines parties du Burkina Faso avec des facteurs pouvant en expliquer la localisation. Outre la poussée générale vers le sud, déjà mentionnée, nous en proposons deux, la proximité de réserves naturelles et de gisements d'or exploités par des mines industrielles ou des orpailleurs.

13 Le premier facteur est manifeste dans les angles nord-est et sud-est du pays, un bon nombre d'actes terroristes se situant dans ou autour de réserves naturelles, où leurs auteurs trouvent refuge. Le second est bien visible au nord où un grand nombre d'actes se situent entre la réserve sylvopastorale et de faune du Sahel, au nord de Dori et un groupe de mines d'or, industrielles ou exploitées par des orpailleurs ${ }^{9}$ : ces dernières semblent favoriser davantage la multiplication des actes terroristes, peut-être car elles sont plus faciles à racketter. Possibilité de refuge et sources de financement se conjuguent donc dans cette zone et facilitent la propagation vers le sud des actes de violence.

Figure 3. Localisation des actes terroristes au Burkina Faso et facteurs explicatifs

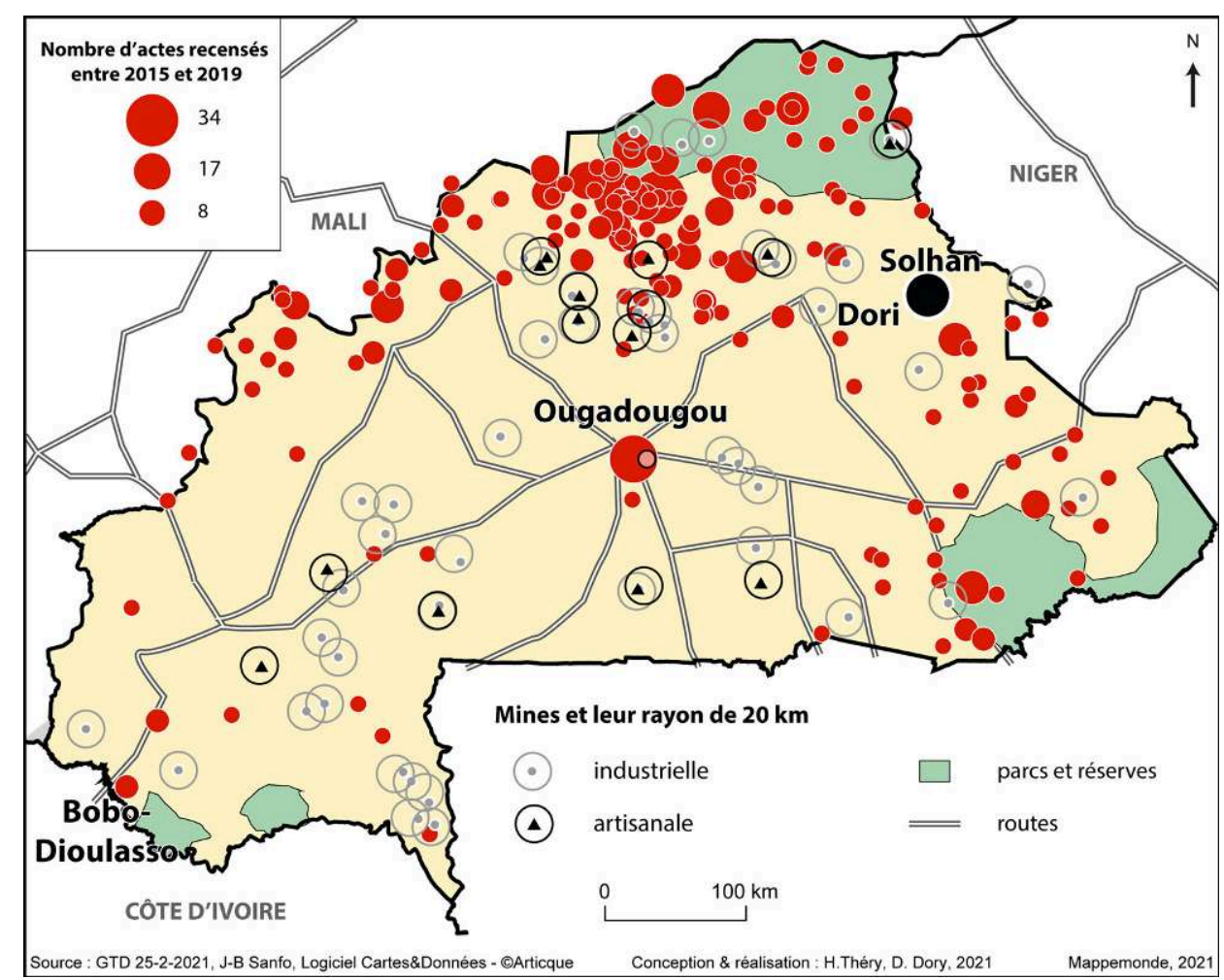

14 Le fait capital qui se dégage de ces cartes est la confirmation d'un mouvement d'ensemble de la dynamique insurrectionnelle vers le sud, conférant au Burkina Faso une importance décisive en tant que zone principale d'expansion de la violence djihadiste dans la région. Et, à partir de ce constat majeur et de la prise en compte de facteurs locaux, il est possible de parvenir à de solides hypothèses prédictives 
concernant de futures attaques. Nous nous dispenserons d'insister sur l'importance géostratégique et pratique de cette conclusion...

\section{NOTES}

1. Parue dans le numéro $34 / 2021$ de Conflits.

2. https://www.revueconflits.com/attentat-solhan-herve-thery-daniel-dory-burkina-faso/

3. Voir, par exemple: Christian Bouquet, «Peut-on parler de "seigneurs de guerre" dans la zone sahélo-saharienne? Entre vernis idéologique et crime organisé », Afrique Contemporaine, $\mathrm{n}^{\circ} 245$, 2013, p. 85-97 ; Héni Nsaibia, Caleb Weiss, “Ansaroul Islam and the Growing Terrorist Insurgency in Burkina Faso", CTC Sentinel, 2018, vol. 11, n 3, p. 21-26.

4. Pour une première approche on peut consulter: Daniel Dory, "Les Terrorism studies à l'heure du bilan ", Sécurité Globale, $n^{\circ} 22,2020$, p. 123-142.

5. On trouvera un aperçu de la bibliographie spécialisée dans: Daniel Dory, "L'analyse géopolitique du terrorisme: conditions théoriques et conceptuelles ", L'Espace Politique, $\mathrm{n}^{\circ} 33$, 2017, en ligne: https://journals.openedition.org/espacepolitique/4482; Daniel Dory, «Le terrorisme comme objet géographique : un état des lieux ", Annales de Géographie, $\mathrm{n}^{\circ}$ 728, 2019, p. 5-36.

6. Par exemple : Olivier Walther, Denis Retaillé, Sahara or Sahel? The fuzzy geography of terrorism in West Africa, CEPS/INSTEAD, Working Paper n²010-35, 2010 ; Clémence Pernin, Houari Sayad, « Le Sahel : terrain de jeu d'al Qaïda au Maghreb Islamique (AQMI) ", Les Cahiers d'Outre-Mer, $\mathrm{n}^{\circ} 255$, 2011, p. 445-446 ; David B. Skillicorn et al. "The Diffusion and Permeability of Political Violence in North and West Africa", Terrorism and Political Violence, 2019 (Preprint).

7. Gary Lafree, Laura Dugan, "Introducing the Global Terrorism Database", Terrorism and Political Violence, vol. 19, $\mathrm{n}^{\circ}$ 2, 2007, p. 181-204. Pour une présentation des bases de données actuellement actives: Neil G. Bowie, "Terrorism Events Data: An Inventory of Databases and Data Sets, 1968-2017", Perspectives on Terrorism, vol. 11, n 4, 2017, p. 50-72.

8. Voir, par exemple, Wesley S. McCann, "Who Said We Where Terrorists? Issues with Terrorism Data and Inclusion Criteria", Studies in Conflict and Terrorism, 2020.

9. Sanfo J.-B. "Cross-Sectional Analysis of the Determinants of Education Demand in Small-Scale Gold Mining Communities in Burkina Faso", Journal of Education and Practice, vol. 10, $\mathrm{n}^{\circ}$ 12, 2019. DOI: $10.7176 / \mathrm{JEP}$

\section{AUTEURS}

\section{HERVÉ THÉRY}

Directeur de recherche émérite au CNRS-Creda Professeur à l'Université de São Paulo (USPPPGH) 


\section{DANIEL DORY}

Maitre de Conférences HDR à l'Université de La Rochelle 